\title{
Quantum field theory of space-like neutrino
}

\author{
Jakub Rembieliński $^{1, \mathrm{a}}{ }_{\mathbb{D}}$, Paweł Caban $^{1, \mathrm{~b}}{ }_{\mathbb{D}}$, Jacek Ciborowski $^{2, \mathrm{c}}$ (D) \\ ${ }^{1}$ Department of Theoretical Physics, Faculty of Physics and Applied Informatics, University of Łódź, Pomorska 149/153, 90-236 Lodz, Poland \\ ${ }^{2}$ Department of Physics, University of Warsaw, Pasteura 5, 02-093 Warsaw, Poland
}

Received: 27 December 2020 / Accepted: 26 July 2021 / Published online: 10 August 2021

(c) The Author(s) 2021

\begin{abstract}
We performed a Lorentz covariant quantization of the spin- $1 / 2$ fermion field assuming the space-like energymomentum dispersion relation. We achieved the task in the following steps: (i) determining the unitary realizations of the inhomogenous Lorentz group in the preferred frame scenario by means of the Wigner-Mackey induction procedure and constructing the Fock space; (ii) formulating the theory in a manifestly covariant way by constructing the field amplitudes according to the Weinberg method; (iii) obtaining the final constraints on the amplitudes by postulating a Dirac-like free field equation. Our theory allows to predict all chiral properties of the neutrinos, preserving the Standard Model dynamics. We discussed the form of the fundamental observables, energy and helicity, and show that nonobservation of the $+\frac{1}{2}$ helicity state of the neutrino and the $-\frac{1}{2}$ helicity state of the antineutrino could be a direct consequence of the "tachyoneity" of neutrinos at the free level. We found that the free field theory of the space-like neutrino is not invariant under the $\mathrm{C}$ and $\mathrm{P}$ transformations separately but is CP-invariant. We calculated and analyzed the electron energy spectrum in tritium decay within the framework of our theory and found an excellent agreement with the recent measurement of KATRIN. In our formalism the questions of negative/imaginary energies and the causality problem does not appear.
\end{abstract}

\section{Introduction}

After nearly 90 years of the neutrino history this particle is still an enigma with a number of unanswered questions in the neutrino physics and the Standard Model. We know that neutrinos oscillate so at last two neutrino generations are massive. Therefore the neutrino field should possess two

\footnotetext{
a e-mail: jaremb@uni.lodz.pl

b e-mail: P.Caban@merlin.phys.uni.lodz.pl (corresponding author)

c e-mail: cib@fuw.edu.pl
}

spin components and consequently neutrinos should be found in two helicity states. However, only the left-handed helicity component of the neutrino and the right-handed of the antineutrino have been observed in experiments. A standard but rather technical explanation of this fact makes use of the see-saw mechanism [1-3]. In contrast, we adopt a hypothesis that the neutrino is a particle satisfying the space-like dispersion relation. This assumption is suggested by a repeating occurrence of negative values for the neutrino mass squared measured in numerous recent tritium-decay experiments [49]. This observation does not make a proof that neutrino is a tachyon because of an insufficient level of confidence of each of these separate results, however, it encourages considering a possible theoretical descriptions of this possibility. Such trials have already been undertaken in the past. A hypothesis that neutrino might be a space-like particle was first discussed by Chodos et al. [10]. Some arguments supporting this proposition were also presented by Giannetto et al. [11]. However, these attempts were unsuccessful due to the fact that the standard relativistic quantum field theory is inapplicable for describing space-like particles, as pointed out by Kamoi and Kamefuchi [12] and Nakanishi [13]. On the other hand, it was shown [14] that an approach involving the notion of a preferred frame (PF) allows to construct a Lorentz-covariant quantum field-theoretical model of a relativistic helicity- $\frac{1}{2}$ fermionic tachyon. In this way one can avoid the fundamental difficulties related to the lack of a finite lower energy limit, appearance of infinite spin multiplets and causal problems appearing in the standard attempts to describe tachyons. In the space-like neutrino case the cosmic neutrino background (CNB) frame, an artefact of the electroweak phase transition [15], is a natural candidate for the preferred frame. In the above framework the $\beta$ decay was considered in [16] and the corresponding decay rate (energy spectrum) for electrons was derived and discussed in the context of the neutrino mass measurement. For other contributions to the tachyonic neutrino hypothesis see [17-24]. 
References to the notion of a PF in the context of the quantum theory are owed to several authors. The historical term of "aether" used in the field-theoretical context by Dirac [25,26] was superseded by that of the PF, as, e.g., in de Broglie-Bohm formulation of quantum mechanics [27,28]. Bell suggested that it would have been helpful to consider a PF at the fundamental level for resolving incompatibilities between special relativity theory and nonlocality of quantum mechanics [29] - an opinion also shared by other authors [30-35]. Let us mention in particular: Lorentz-violating extensions of the Standard Model [36-39], approaches to classical and quantum gravity like the Einstein's aether [40] and HořavaLifshitz theories of gravity [41] (including vacuum solutions in this model [42]), and the so-called doubly special relativity theories [43], characterized by modified dispersion relations, common for the Lorentz violating models. In almost all of the above theories specific physical effects are predicted, of magnitude usually suppressed by a power of the Planck scale, like, e.g., vacuum birefringence [36-40,43]. This brief outlook demonstrates that the concept of a PF has been frequently referred to in the context of Lorentz symmetry violation within numerous contemporary theories.

It is important to stress that if tachyons exist, one could, in principle, consider synchronizing distant clocks in almost absolute way (instantaneous synchronization) in the limit of zero energy (infinite velocity) if they interact with matter with finite probability in these conditions. In consequence, one has a possibility of introducing absolute time between observers. The only way to reconcile this implication with the Lorentz symmetry lies in an assumption of existence of a preferred frame [14]. We discuss this point in Sect. 2.

In the present paper we formulate a fully consistent quantum field theory of the space-like neutrino with both onehalf helicity components under the assumption of the existence of the preferred frame. In our approach the states of tachyonic field excitations observed from an arbitrary inertial frame should depend on the PF four-velocity as seen from the observer frame. All other physical fields/states are simply unaffected by the PF. This means that the PF is related with the tachyonic sector only. As we will see the preferred frame concept is crucial for the successful construction of a viable theory of fermionic tachyons enabling to eliminate the issue of negative/imaginary energies and causality problems as well as to perform the field quantization procedure in arbitrary inertial frames. At the interaction level we obtain the anomaly free, perturbatively renormalizable variant of the electroweak model. We construct this theory in few steps. Firstly, in Sect. 2 we construct the one-particle phase space and discuss its Lorentz covariance as well as the issue of imaginary energies, stability of the theory and causality. Next, in Sect. 3, we determine the unitary realizations of the inhomogenous Lorentz group under the condition of existence of an inertial preferred frame. We do this by means of the Wigner-Mackey induction procedure [44]. Secondly, in Sect. 4, we construct the Fock space by generation of the multiparticle basis states from the Lorentz invariant vacuum state by using the corresponding algebra of creation/annihilation operators. This quantization procedure is formulated for all inertial frames. Thirdly, in Sect. 5, we formulate the theory in a manifestly covariant way by constructing the field amplitudes according to the Weinberg method [45]. Fourthly, in Sect. 6, we obtain the final constraints on the amplitudes by postulating a Dirac-like free field equation determining the free space-like neutrino field completely. Next, in Sect. 7 we show that the amplitudes of the space-like neutrino field have properties which are consistent with the high energy chiral properties of the neutrino observed in reality. On the other hand, we predict that at very low neutrino energies, both helicities should be observed. We discuss the form of the fundamental observables, namely energy and helicity. Sect. 8 is devoted to the discussion of the propagator of the spacelike particles. In Sect. 9 we find that the free field theory of the space-like neutrino is not invariant under the parity and charge conjugation transformations separately, however, it is invariant under the CP transformation. Finally, in Sect. 11 we construct the anomaly free variant of the electroweak model and calculate and analyze the $\beta$ decay rate (electron energy spectrum) in this context.

Because the tachyon kinematics in the preferred frame scenario as well as the construction of the corresponding quantum field theory is not widely known, we present our approach with necessary details, restricting ourselves to one neutrino generation for simplicity.

\section{Tachyon kinematics in the preferred frame scenario}

Tachyon is a particle with a space-like four-momentum $k^{\mu}$, satisfying the following Lorentz-covariant dispersion relation

$k^{\mu} k_{\mu}=k^{0^{2}}-\mathbf{k}^{2}=-\kappa^{2}$,

where $\kappa$ denotes the "tachyonic mass" $(\kappa>0)$. However, $\kappa$ can be also viewed as a residual momentum of the tachyon in the limit of zero energy. Eq. (1) defines a one sheet hyperboloid where energy $k^{0}$ takes the values from minus to plus infinity. Lorentz transformations relating inertial frames can transform positive $k^{0}$ to any negative value. Hence, this causes the vacuum instability on the quantum level, i.e., possibility of a spontaneous creation from the vacuum of pairs of particles with the total four-momentum equal to zero. Below we show that this problem can be resolved in the preferred frame scenario. Let us denote the inertial observer's frame by $\Sigma_{u}$. From his point of view the PF, $\Sigma_{P F}$, moves with a 
constant four-velocity $u^{\mu}$ satisfying

$u^{0^{2}}-\mathbf{u}^{2}=1$.

Thus for observers stationary in the PF, its four-velocity is given by $u_{P F}=(1,0,0,0)$. The PF three-velocity (in units of $c$ ) is given by $\mathbf{V}=\mathbf{u} / u^{0}$ so $u^{0}=1 / \sqrt{1-\mathbf{V}^{2}}$ is the Lorentz factor of the PF. Now, using the four-momentum $k^{\mu}$ of the tachyon and the four-velocity $u^{\mu}$ we can construct a Lorentz invariant

$q=u k=u^{\mu} k_{\mu}=u^{0} k^{0}-\mathbf{u} \cdot \mathbf{k}$

equal to the tachyonic energy measured in the preferred frame. Hence, the physically acceptable four-momenta $k^{\mu}$ are bounded by the Lorentz covariant condition

$q>0$

guaranting nonnegativity of energy in the preferred frame. We see from Eq. (3) that only in the preferred frame, $\Sigma_{P F}$, tachyons have always non negative energies. Indeed, applying the Lorentz boost transformation one can also obtain kinematical states of the tachyon with negative energies in other inertial frames (but still with a lower bound set by Eq. (4)). The explicit form of the energy and the value of the momentum obtained from the relations $(1,2,3,4)$ in an arbitrary inertial frame $\Sigma_{u}$ is the following

$$
\begin{aligned}
k^{0} & =\frac{q u^{0}+\cos \theta \sqrt{\left(u^{0}\right)^{2}-1} \sqrt{q^{2}+\kappa^{2}\left[\cos ^{2} \theta+\left(u^{0}\right)^{2} \sin ^{2} \theta\right]}}{\cos ^{2} \theta+\left(u^{0}\right)^{2} \sin ^{2} \theta}, \\
|\mathbf{k}| \equiv \omega(q, u, \theta) & =\frac{q \cos \theta \sqrt{\left(u^{0}\right)^{2}-1}+u^{0} \sqrt{q^{2}+\kappa^{2}\left[\cos ^{2} \theta+\left(u^{0}\right)^{2} \sin ^{2} \theta\right]}}{\cos ^{2} \theta+\left(u^{0}\right)^{2} \sin ^{2} \theta},
\end{aligned}
$$

where $q>0, u^{0}=1 / \sqrt{1-\mathbf{V}^{2}}, \theta$ is the angle between the momentum, $\mathbf{k}$, and the PF velocity, $\mathbf{V}$. Thus, the energy bound in an arbitrary frame $\Sigma_{u}$ has the following form

$k^{0}>\frac{\kappa|\mathbf{V}| \cos \theta}{\sqrt{1-(|\mathbf{V}| \cos \theta)^{2}}}$.

We introduce an invariant measure respecting the dispersion relation (1) and the covariant condition (4):

$$
\begin{aligned}
d \mu(k, u) & =d^{4} k \delta\left(k^{2}+\kappa^{2}\right) \Theta(u k) \\
& =\frac{1}{2 u^{0}}|\mathbf{k}| \Theta(q) d q d \Omega,
\end{aligned}
$$

(see Appendix A), where $d \Omega$ is the solid angle differential related to the neutrino momentum $\mathbf{k},|\mathbf{k}|$ is given in Eq. (6) and $\Theta$ is the Heaviside Theta function.

Now, we comment common objections against the tachyonic theories in the context of the formalism introduced above.

\subsection{Problem of negative energies}

Negative energies can formally appear in the standard description of tachyons for the reason that covariant lower bound of energy does not exist in this case. In contrast, existence of a preferred frame allows to define the Lorentz covariant condition (4), constituting the lower bound of energy in each inertial frame, eliminating possibility of the kinematical instability. Indeed, if the four-momentum $k^{\mu}$ belongs to the upper half of the one-sheet hyperboloid, i.e., it satisfies the inequality $q=u k>0$ then $-k^{\mu}$ must belong to the lower part of hyperboloid because $(-u k)=-q<0$ does not satisfy the condition (4). Therefore, the condition (4) eliminates the possibility of the kinematical instability. We note that this condition is analogous to choosing the upper energymomentum cone for massless particles as the physical one.

\subsection{Problem of imaginary energies}

This is rather fictitious problem in the case of particles satisfying the dispersion relation (1). It is rooted to the fact that Eq. (1) implies $k^{0} \sim \sqrt{\mathbf{k}^{2}-\kappa^{2}}$ so $k^{0}$ can be in principle imaginary for $\mathbf{k}^{2}<\kappa^{2}$ and we can expect exponentially divergent trajectories in that case. However this is impossible for tachyons on the mass shell, i.e., satisfying the dispersion relation (1) because in that case the inequality $|\mathbf{k}|>\kappa$ must hold for physical momenta. In our formalism this is guaranteed by the choice of the invariant measure (8) which vanishes inside the sphere defined by inequality $\mathbf{k}^{2}=\kappa^{2}$. Absence of the imaginary eigenvalues of the energy operator is also evident in the explicit formulation of our theory in Sects. 4-6.

\subsection{Problem of non-causal behavior}

The notion of causality is inseparably related to the definition of the coordinate time. In the standard relativistic theories time is identified with the zeroth coordinate in the Minkowski space-time. Consequently, for a space-like separation of events, the Lorentz transformations can change their time ordering. However, existence of a preferred frame provides a solution of this problem. Namely, we can introduce another, Lorentz invariant, dynamical parameter, $T:=u_{\mu} x^{\mu}$. Notice, that this parameter allows a Lorentz invariant $T$-time ordering is in this case Lorentz invariant. In the PF, $T=x_{P F}^{0}$, i.e., $t_{P F}=T$ so it is equal to the Einsteinian time (in the $c$ units). In an arbitrary frame $\Sigma_{u}$ from the definition of $T$, we obtain $\tau \equiv \frac{t_{P F}}{u^{0}}=t-\mathbf{V} \cdot \mathbf{x}$ where $\mathbf{V}$ is the defined above velocity of the preferred frame, i.e., $\mathbf{V}=\mathbf{u} / u^{0}$. The coordinate time redefinition $\tau=t-\mathbf{V} \cdot \mathbf{x}$ between the Einsteinian time $t$ and the time $\tau$ is simply the admissible change of clock synchronization [14]. For a stationary observer, in each fixed point $(d \mathbf{x}=0) d t=d \tau$, i.e., the flow of the time 
is the same. However, it follows from the time redefinition formula that $\frac{d \tau}{d t}=1-\mathbf{V} \cdot \boldsymbol{\vartheta}$, where the velocity $\vartheta=\frac{d \mathbf{x}}{d t}$. Notice that for subluminal or luminal motion $(|\vartheta| \leq 1)$ the derivative $d \tau / d t$ is always nonnegative, i.e., the arrows of the Einsteinian time $t$ and the time $\tau$ are the same (causality, i.e., sequence of events is the same) . However, for superluminal motion $(|\vartheta|>1)$, the derivative $d \tau / d t$ can change sign to negative because the scalar product $\mathbf{V} \cdot \boldsymbol{\vartheta}$ can, in some velocity configurations, exceed 1 . Because $d \tau$ is always positive (the arrow of time $t_{P F}$ is fixed) then $d t$ must change the sign, which corresponds to the indeterminacy of the causal relation in the Einsteinian synchronization scheme for velocities higher than the light velocity while, in terms of the time $\tau$, it is determined for all velocities. Moreover, the time$\tau$ synchronization satisfies the crucial physical requirement - the average value of the light velocity over closed paths is frame independent and equal to $c$. For more information on the issue of clock synchronization in the special relativity see [46-48]. Summarizing, only the $\tau$ synchronization is adequate to determine uniquely the sequence of events (causality) for phenomena with participation of tachyons in the presence of the PF or equivalently, causality should be referred to observers stationary in the PF.

\section{Space of states of the space-like neutrino}

The condition sine qua non to apply the tachyonic hypothesis to neutrino physics is to construct a quantum field theory of space-like fields. Indeed, the standard relativistic field theory is inapplicable to this case because of two reasons: Firstly, the Wigner little group of the space-like four-momentum of the tachyon is the noncompact $\mathrm{SO}(2,1)$ group, so its unitary representations are scalar or infinite-dimensional. Consequently, the spin multiplets are either one-dimensional or infinitedimensional. Therefore, a spin- $\frac{1}{2}$ neutrino cannot be identified with such representations. Secondly, if we go around the above problem and try describing the tachyonic neutrino with the help of the bi-spinor nonunitary representation by identifying the Lagrange density appropriate to the spacelike dispersion relation as is done, e.g., in [10], we evidently loose unitarity, i.e., the probabilistic character of the quantum description. However, in the scenario with a PF, these difficulties do not arise.

As was stated above, a reasonable description of tachyons needs the presence of a preferred frame. Consequently, the tachyonic neutrino basis states should be dependent not only on the space-like four-momentum, $k^{\mu}$, but also on the PF four-velocity, $u^{\mu}$. Our aim is to apply the Wigner-Mackey induction procedure in this case. Hereafter we will denote eigenvectors of the four-momentum operator as $|k, u, \lambda\rangle$, where $\lambda$ is identified with the neutrino/antineutrino helicity. Now, an observer in an arbitrary reference frame $\Sigma_{u}$ has at his disposal the Hilbert space $\mathcal{H}_{u}$ of neutrino states. The family of Hilbert spaces $\mathcal{H}_{u}$ form a bundle corresponding to the bundle of inertial frames $\Sigma_{u}$, i.e., to the quotient manifold $\mathrm{SO}(1,3) / \mathrm{SO}(3) \sim \mathbb{R}^{3}$ as the base space and with $\mathcal{H}_{u}$ as fibers. To apply the Wigner-Mackey construction to this case we should find the little group of the pair of four-vectors $(k, u)$ determining the neutrino state. To do this we transform the pair $(k, u)$ to the preferred frame by action of the boost $L_{u}^{-1}$ to obtain the pair $\left(L_{u}^{-1} k=k^{\prime}, L_{u}^{-1} u=u_{P F}\right)$. Next we rotate $k^{\prime}$ with the help of the rotation $R_{\mathrm{n}}^{-1}$ to the $z$-axis in order to obtain $\tilde{k}=\left(L_{u} R_{\mathbf{n}}\right)^{-1} k=\left(q ; 0,0, \sqrt{q^{2}+\kappa^{2}}\right)$ while $u_{P F}$ is left unchanged (for the explicit form of $L_{u}$ and $R_{\mathbf{n}}$ see Appendix B). Thus, the pair $(k, u)$ can be obtained from the standard pair $\left(\tilde{k}, u_{P F}\right)$ by a sequence of Lorentz transformations $L_{u} R_{\mathbf{n}}$, i.e.,

$(k, u)=L_{u} R_{\mathbf{n}}\left(\tilde{k}, u_{P F}\right)$

provided the unit vector $\mathbf{n}$ is equal to

$\mathbf{n}=\mathbf{n}(k, u)=\frac{1}{\sqrt{q^{2}+\kappa^{2}}}\left(\mathbf{k}-\frac{q+k^{0}}{1+u^{0}} \mathbf{u}\right)$,

where, by means of Eqs. (3) and (4), $k^{0}=(q+\mathbf{u} \cdot \mathbf{k}) / u^{0}$ and $q>0$. It is obvious that the orthogonal group $\mathrm{SO}(2)$ is the stability group of the pair $\left(\tilde{k}, u_{P F}\right)$. Therefore, irreducible unitary orbits of the inhomogeneous Lorentz group should be induced from the unitary irreducible representations of $\mathrm{SO}(2)$, i.e., from the one-dimensional representation of the $\mathrm{U}(1)$ group. Applying the Wigner-Mackey procedure to the basis vectors $|k, u, \lambda\rangle, \lambda \in \mathbb{R}$, in the manifold of Hilbert spaces $\mathcal{H}_{u}$ we obtain a result that the unitary action of the Lorentz group is of the form

$U(\Lambda)|k, u, \lambda\rangle=e^{i \lambda \varphi(\Lambda, k, u)}|\Lambda k, \Lambda u, \lambda\rangle$,

where $e^{i \varphi(\Lambda, k, u)}$ is the phase factor corresponding to the Wigner rotation

$W(k, u, \Lambda)=\left(L_{\Lambda u} R_{\mathbf{n}(\Lambda k, \Lambda u)}\right)^{-1} \Lambda L_{u} R_{\mathbf{n}(k, u)}$.

The standard arguments lead to integer or half-integer values of $\lambda$. In the case of the tachyonic neutrino we restrict further considerations to the values $\lambda= \pm \frac{1}{2}$.

Moreover, in each fixed space $\mathcal{H}_{u}$ we adopt the following Lorentz covariant normalization for the momentum eigenstates for neutrinos and antineutrinos

$\left\langle k, u, \lambda \mid k^{\prime}, u, \lambda^{\prime}\right\rangle=\frac{1}{|\mathbf{k}|} 2 u^{0} \delta_{\lambda \lambda^{\prime}} \delta\left(q-q^{\prime}\right) \delta\left(\hat{\mathbf{k}}-\hat{\mathbf{k}}^{\prime}\right)$,

where $\hat{\mathbf{k}}$ denotes the unit vector $\mathbf{k} /|\mathbf{k}|$ and $\delta\left(\hat{\mathbf{k}}-\hat{\mathbf{k}}^{\prime}\right)$ is the spherical Dirac delta (see Appendix A). Notice that it is convenient to choose the $z$-axis in the direction of the preferred frame velocity $\mathbf{V}$, the angle $\theta$ is the polar angle of $\hat{\mathbf{k}}$ in such a case.

Therefore, the one-particle space of tachyonic states is a direct sum of one particle tachyonic neutrino $\oplus$ antineutrino 
space (of course neutrino and antineutrino spaces are mutually orthogonal). The completeness relation in this space has the form

$\sum_{\lambda} \int d \mu(k, u)\left(|k, u, \lambda\rangle_{\mathcal{V}}\langle k, u, \lambda|+| k, u, \lambda\rangle_{\bar{\nu}}\langle k, u, \lambda|\right)=I$,

where the Lorentz invariant measure $d \mu$ is defined in Eq. (8). We prove this relation explicitly in Appendix A.

\section{Fock space of the space-like neutrino}

We construct a free field theory of the tachyonic neutrino in the preferred frame scenario, in close analogy with the standard formalism, restricted to a single neutrino generation for simplicity. Because an irreducible realization of the Lorentz group is fixed in our case by the choice of $\lambda$, the basis vectors $|k, u, \lambda\rangle$ are obtained by the action of the neutrino creation operators $a_{\lambda}^{\dagger}(k, u)$ and antineutrino creation operators $b_{\lambda}^{\dagger}(k, u)$ on the normalized to the unity vacuum vector $|0\rangle$ defined by the standard conditions

$a_{\lambda}(k, u)|0\rangle=b_{\lambda}(k, u)|0\rangle=0$.

Thus

$\begin{aligned}|k, u, \lambda\rangle_{v} & =a_{\lambda}^{\dagger}(k, u)|0\rangle, \\ |k, u, \lambda\rangle_{\bar{v}} & =b_{\lambda}^{\dagger}(k, u)|0\rangle .\end{aligned}$

The above vectors respect the normalization (13) provided the creation and annihilation operators satisfy, for each fixed $u$, the following anti-commutation canonical relations

$$
\begin{aligned}
& \left\{a_{\lambda}(k, u), a_{\sigma}^{\dagger}\left(k^{\prime}, u\right)\right\} \\
& \quad=\frac{1}{\omega(q, u, \theta)} 2 u^{0} \delta\left(q-q^{\prime}\right) \delta\left(\hat{\mathbf{k}}-\hat{\mathbf{k}}^{\prime}\right) \delta_{\lambda \sigma}, \\
& \left\{b_{\lambda}(k, u), b_{\sigma}^{\dagger}\left(k^{\prime}, u\right)\right\} \\
& \quad=\frac{1}{\omega(q, u, \theta)} 2 u^{0} \delta\left(q-q^{\prime}\right) \delta\left(\hat{\mathbf{k}}-\hat{\mathbf{k}}^{\prime}\right) \delta_{\lambda \sigma} .
\end{aligned}
$$

The remaining anti-commutators vanish.

To reproduce formula (11), creation operators should transform under the action of the Lorentz group according to the law

$$
\begin{aligned}
& U(\Lambda) a_{\lambda}^{\dagger}(k, u) U(\Lambda)^{\dagger}=e^{i \lambda \varphi(\Lambda, k, u)} a_{\lambda}^{\dagger}(\Lambda k, \Lambda u), \\
& U(\Lambda) b_{\lambda}^{\dagger}(k, u) U(\Lambda)^{\dagger}=e^{i \lambda \varphi(\Lambda, k, u)} b_{\lambda}^{\dagger}(\Lambda k, \Lambda u) .
\end{aligned}
$$

The corresponding Fock space of multiparticle states can be now defined in a standard way by a successive action of creation operators on the vacuum state. At this stage, given the Lorentz invariant measure, the space of states and the corresponding transformation rules, we are ready to define the fundamental observables. The helicity operator $\hat{\lambda}$ is defined as

$$
\begin{gathered}
\hat{\lambda}(u)=\sum_{\lambda} \int d \mu(k, u) \lambda\left(a_{\lambda}^{\dagger}(k, u) a_{\lambda}(k, u)\right. \\
\left.+b_{\lambda}^{\dagger}(k, u) b_{\lambda}(k, u)\right)
\end{gathered}
$$

and by means of (18-19) it satisfies

$$
\begin{aligned}
& {\left[\hat{\lambda}, a_{\lambda}^{\dagger}(k, u)\right]=\lambda a_{\lambda}^{\dagger}(k, u),} \\
& {\left[\hat{\lambda}, b_{\lambda}^{\dagger}(k, u)\right]=\lambda b_{\lambda}^{\dagger}(k, u) .}
\end{aligned}
$$

Similarly, we define the covariant four-momentum operator $\hat{P}_{\mu}$ by the standard formula

$$
\begin{aligned}
\hat{P}_{\mu}(u)= & \int d \mu(k, u) k_{\mu} \sum_{\lambda}\left(a_{\lambda}^{\dagger}(k, u) a_{\lambda}(k, u)\right. \\
& \left.+b_{\lambda}^{\dagger}(k, u) b_{\lambda}(k, u)\right)
\end{aligned}
$$

which implies, that

$$
\begin{aligned}
& {\left[\hat{P}_{\mu}, a_{\lambda}^{\dagger}(k, u)\right]=k_{\mu} a_{\lambda}^{\dagger}(k, u),} \\
& {\left[\hat{P}_{\mu}, b_{\lambda}^{\dagger}(k, u)\right]=k_{\mu} b_{\lambda}^{\dagger}(k, u) .}
\end{aligned}
$$

\section{Manifestly covariant formulation}

The neutrino field $v(x, u)$ is defined as the Dirac bispinor operator of the form

$$
\begin{aligned}
v_{\alpha}(x, u)= & \frac{1}{(2 \pi)^{3 / 2}} \sum_{\lambda} \int d \mu(k, u)\left[e^{i k x} \mathrm{v}_{\alpha \lambda}(k, u) b_{\lambda}^{\dagger}(k, u)\right. \\
& \left.+e^{-i k x} \mathrm{u}_{\alpha \lambda}(k, u) a_{\lambda}(k, u)\right],
\end{aligned}
$$

with the standard manifestly covariant transformation rule

$U(\Lambda) v(x, u) U(\Lambda)^{\dagger}=S\left(\Lambda^{-1}\right) v(\Lambda x, \Lambda u)$,

where $S(\Lambda)$ belongs to the representation $D^{\left(\frac{1}{2}, 0\right)} \oplus D^{\left(0, \frac{1}{2}\right)}$ of the homogenous Lorentz group.

In order to fulfill the transformation law (29), the amplitudes $\mathrm{v}_{\lambda}(k, u)$ and $\mathrm{u}_{\lambda}(k, u)$, must satisfy the Weinberg consistency conditions obtained with the use of Eqs. $(20,21,28)$, namely

$\mathrm{u}_{\lambda}(\Lambda k, \Lambda u)=S(\Lambda) \mathbf{u}_{\lambda}(k, u) e^{-i \lambda \varphi(\Lambda, k, u)}$,

$\mathrm{v}_{\lambda}(\Lambda k, \Lambda u)=S(\Lambda) \mathrm{v}_{\lambda}(k, u) e^{i \lambda \varphi(\Lambda, k, u)}$.

In the following we choose the Weyl bi-spinor representation of the Lorentz group in the form

$S(\Lambda(A))=\left(\begin{array}{cc}A & 0 \\ 0 & A^{\dagger-1}\end{array}\right)$, 
where the matrices $A$ belong to the $\mathrm{SL}(2, \mathbb{C})$ group. The corresponding representation of the $\gamma$ matrices is the following

$\gamma^{0}=\left(\begin{array}{cc}0 & I \\ I & 0\end{array}\right), \quad \gamma^{k}=\left(\begin{array}{cc}0 & \sigma^{k} \\ -\sigma^{k} & 0\end{array}\right)$,

$\gamma^{5}=\gamma_{5}=i \gamma^{0} \gamma^{1} \gamma^{2} \gamma^{3}=\left(\begin{array}{cc}-I & 0 \\ 0 & I\end{array}\right)$,

where $\sigma^{k}$ are the standard Pauli matrices. Note that the Pauli matrices are contravariant. In the Weyl representation the neutrino field and the amplitudes $\mathrm{v}(k, u)=\left[\mathrm{v}_{\alpha \lambda}(k, u)\right]$ and $\mathrm{u}(k, u)=\left[\mathrm{u}_{\alpha \lambda}(k, u)\right]$ admit the following chiral decompositions

$\mathrm{u}(k, u)=\left(\begin{array}{l}\mathrm{u}_{L}(k, u) \\ \mathrm{u}_{R}(k, u)\end{array}\right), \quad \mathrm{v}(k, u)=\left(\begin{array}{c}\mathrm{v}_{L}(k, u) \\ \mathrm{v}_{R}(k, u)\end{array}\right)$

corresponding to the chiral projections $P_{L / R}=\frac{1}{2}\left(I \mp \gamma^{5}\right)$.

By means of Eqs. $(9,12,30,31,32)$ we obtain

$\mathrm{u}(k, u)=S\left(L_{u} R_{\mathbf{n}}\right) \mathrm{u}\left(\tilde{k}, u_{P F}\right)$,

$\mathrm{v}(k, u)=S\left(L_{u} R_{\mathbf{n}}\right) \mathrm{v}\left(\tilde{k}, u_{P F}\right)$,

where

$S\left(L_{u} R_{\mathbf{n}}\right)=\left(\begin{array}{cc}\mathbb{L}_{u} \mathbb{U}_{\mathbf{n}} & 0 \\ 0 & \mathbb{L}_{u^{\pi}} \mathbb{U}_{\mathbf{n}}\end{array}\right)$,

and $\mathbb{L}_{u}$ is the Lorentz boost matrix representing $L_{u}$ in the left-handed spinor space, $\mathbb{L}_{u^{\pi}}$ is the boost acting in the righthanded spinor space, $\pi$ denotes the parity operation on fourvectors whereas $\mathbb{U}_{\mathbf{n}}$ represents the rotation $R_{\mathbf{n}}$. Explicitly

$$
\begin{aligned}
\mathbb{L}_{u} & =\frac{1}{\sqrt{2\left(1+u^{0}\right)}}\left(\left(1+u^{0}\right) I-\mathbf{u} \cdot \boldsymbol{\sigma}\right), \\
\mathbb{L}_{u^{\pi}} & =\mathbb{L}_{u}^{-1}=\frac{1}{\sqrt{2\left(1+u^{0}\right)}}\left(\left(1+u^{0}\right) I+\mathbf{u} \cdot \boldsymbol{\sigma}\right), \\
\mathbb{U}_{\mathbf{n}} & =\frac{1}{\sqrt{2\left(1+n^{3}\right)}}\left(\begin{array}{cc}
1+n^{3} & -n^{1}+i n^{2} \\
n^{1}+i n^{2} & 1+n^{3}
\end{array}\right)
\end{aligned}
$$

and the unit vector $\mathbf{n}(k, u)$ is defined in Eq. (10).

Now, choosing $k=\tilde{k}, u=u_{P F}$ in $(30,31)$ and $\Lambda$ as the stability group element of this pair, i.e., $\Lambda=R_{z}(\phi)$, where $R_{z}(\phi)$ is rotation around the third axis represented, according to (32), by the matrix

$S\left(R_{z}(\phi)\right)=\left(\begin{array}{cc}\mathbb{U}_{z}(\phi) & 0 \\ 0 & \mathbb{U}_{z}(\phi)\end{array}\right)$

with

$$
\mathbb{U}_{z}(\phi)=\left(\begin{array}{cc}
e^{i \phi / 2} & 0 \\
0 & e^{-i \phi / 2}
\end{array}\right)
$$

and noting that from (12) the Wigner phase is given by $\varphi\left(R_{z}(\phi), \tilde{k}, u_{P F}\right)=\phi$ in this case, we obtain the follow- ing conditions for the chiral amplitudes

$$
\begin{aligned}
\mathbb{U}_{z}(\phi) \tilde{\mathrm{u}}_{\lambda_{L / R}}\left(\tilde{k}, u_{P F}\right) e^{-i \lambda \phi} & =\tilde{\mathrm{u}}_{\lambda_{L / R}}\left(\tilde{k}, u_{P F}\right), \\
\mathbb{U}_{z}(\phi) \tilde{\mathrm{v}}_{\lambda_{L / R}}\left(\tilde{k}, u_{P F}\right) e^{i \lambda \phi} & =\tilde{\mathrm{v}}_{\lambda_{L / R}}\left(\tilde{k}, u_{P F}\right),
\end{aligned}
$$

leading to

$$
\begin{gathered}
\tilde{\mathrm{u}}_{-1 / 2_{L / R}}=c_{L / R}\left(\begin{array}{l}
0 \\
1
\end{array}\right), \quad \tilde{\mathrm{u}}_{1 / 2_{L / R}}=c_{L / R}^{\prime}\left(\begin{array}{l}
1 \\
0
\end{array}\right), \\
\tilde{\mathrm{v}}_{1 / 2_{L / R}}=d_{L / R}\left(\begin{array}{l}
0 \\
1
\end{array}\right), \quad \tilde{\mathrm{v}}_{-1 / 2_{L / R}}=d_{L / R}^{\prime}\left(\begin{array}{l}
1 \\
0
\end{array}\right) .
\end{gathered}
$$

In order to determine coefficients $c_{L / R}, c_{L / R}^{\prime}, d_{L / R}, d_{L / R}^{\prime}$, we must use a manifestly covariant first order differential equation connecting the left and right handed chiral components of the neutrino field. This equation must be consistent with the above field construction procedure.

\section{Dirac-like equation}

Now, we introduce an analog of the Dirac equation in our formalism to conclude defining the free dynamics of the tachyonic neutrino field. A variety of the Lorentz covariant Diraclike equations for spin- $\frac{1}{2}$ tachyons was discussed in Ref. [14], however, only two equations satisfy the CPT invariance. Here we adopt the simplest of them, namely

$\left(\gamma^{5} \gamma^{\mu} i \partial_{\mu}-\kappa\right) v(x, u)=0$.

This equation was first introduced by Tanaka [49] while the corresponding Lagrangian was proposed by Chodos et al. [10].

Equation (48) implies the space-like dispersion relation for $k_{\mu}$ and fixes interrelations between left and right components of the neutrino field. We use this equation for the free field $v(x, u)$ introduced in Eq. (28) in the context of our approach to determine the coefficients $c_{L / R}, c_{L / R}^{\prime}, d_{L / R}$, $d_{L / R}^{\prime}$ of field amplitudes in Eqs. $(46,47)$. Thus we obtain the following equations for the amplitudes

$\left(\gamma^{5} \gamma^{\mu} k_{\mu}-\kappa\right) \mathrm{u}_{\lambda}(k, u)=0$,
$\left(\gamma^{5} \gamma^{\mu} k_{\mu}+\kappa\right) \mathrm{v}_{\lambda}(k, u)=0$,

where $k_{\mu}$ satisfies the covariant conditions (1) and (4).

For the chiral amplitudes, the above equations imply

$\mathrm{u}_{\lambda L}(k, u)=-\frac{1}{\kappa}\left(k^{0} I-\mathbf{k} \cdot \boldsymbol{\sigma}\right) \mathrm{u}_{\lambda R}(k, u)$,

$\mathrm{v}_{\lambda L}(k, u)=\frac{1}{\kappa}\left(k^{0} I-\mathbf{k} \cdot \boldsymbol{\sigma}\right) \mathrm{v}_{\lambda R}(k, u)$,

and analogously for the space inverted pair. These equations allow to determine the values of the coefficients $c_{L / R}, c_{L / R}^{\prime}$, 
$d_{L / R}, d_{L / R}^{\prime}$ in Eqs. $(46,47)$ for $k=\tilde{k}$ and $u=u_{P F}$. Taking into account (36) and (37), we obtain the final form of the normalized amplitudes in an arbitrary frame $\Sigma_{u}$ :

$$
\begin{aligned}
& \mathbf{u}_{1 / 2}(k, u)=\frac{1}{\sqrt{2}}\left(\begin{array}{l}
\sqrt{-q+\sqrt{q^{2}+\kappa^{2}}} \mathbb{L}_{u} \mathbb{U}_{\mathbf{n}}\left(\begin{array}{l}
1 \\
0
\end{array}\right) \\
\sqrt{q+\sqrt{q^{2}+\kappa^{2}}} \mathbb{L}_{u^{\pi}} \mathbb{U}_{\mathbf{n}}\left(\begin{array}{l}
1 \\
0
\end{array}\right)
\end{array}\right), \\
& \mathrm{u}_{-1 / 2}(k, u)=\frac{1}{\sqrt{2}}\left(\begin{array}{c}
\sqrt{q+\sqrt{q^{2}+\kappa^{2}}} \mathbb{L}_{u} \mathbb{U}_{\mathbf{n}}\left(\begin{array}{l}
0 \\
1
\end{array}\right) \\
-\sqrt{-q+\sqrt{q^{2}+\kappa^{2}}} \mathbb{L}_{u^{\pi}} \mathbb{U}_{\mathbf{n}}\left(\begin{array}{l}
0 \\
1
\end{array}\right)
\end{array}\right) \text {, } \\
& \mathrm{V}_{1 / 2}(k, u)=\frac{1}{\sqrt{2}}\left(\begin{array}{c}
\sqrt{q+\sqrt{q^{2}+\kappa^{2}}} \mathbb{L}_{u} \mathbb{U}_{\mathbf{n}}\left(\begin{array}{l}
0 \\
1
\end{array}\right) \\
\sqrt{-q+\sqrt{q^{2}+\kappa^{2}}} \mathbb{L}_{u^{\pi}} \mathbb{U}_{\mathbf{n}}\left(\begin{array}{l}
0 \\
1
\end{array}\right)
\end{array}\right), \\
& \mathrm{V}_{-1 / 2}(k, u)=\frac{1}{\sqrt{2}}\left(\begin{array}{l}
\sqrt{-q+\sqrt{q^{2}+\kappa^{2}}} \mathbb{L}_{u} \mathbb{U}_{\mathbf{n}}\left(\begin{array}{l}
1 \\
0
\end{array}\right) \\
-\sqrt{q+\sqrt{q^{2}+\kappa^{2}}} \mathbb{L}_{u^{\pi}} \mathbb{U}_{\mathbf{n}}\left(\begin{array}{l}
1 \\
0
\end{array}\right)
\end{array}\right) \text {, }
\end{aligned}
$$

where $\mathbb{L}_{u}$ and $\mathbb{U}_{\mathbf{n}}$ are given by Eqs. (39) and (41), respectively, while $q$ and $\mathbf{n}$ are functions of $k$ and $u$, defined in Eqs. (3) and (10).

Using $(51,52)$ and the appropriate formulas found in Appendix B one can easily check by means of Eqs. (1, 2, $3,4)$, that the amplitudes $(53,54,55,56)$ fulfill Eqs. $(49,50)$, i.e., the field $v(x, u)$ satisfies Eq. (48). The scalar products of amplitudes and the polarization operators are collected in Appendix C.

Dependence of the amplitudes $(53,54,55,56)$ on $q$ is such that for $q \gg \kappa$ the following chiral amplitudes vanish to high accuracy for each $u_{\mu}$

$\mathrm{u}_{(1 / 2) L}(k, u) \rightarrow 0, \quad \mathrm{u}_{(-1 / 2) R}(k, u) \rightarrow 0$,

$\mathrm{v}_{(1 / 2) R}(k, u) \rightarrow 0, \quad \mathrm{v}_{(-1 / 2) L}(k, u) \rightarrow 0$,

from which the following limiting forms are obtained for $q \gg \kappa$

$\mathrm{u}_{-1 / 2} \rightarrow \frac{1}{2}\left(1-\gamma^{5}\right) \mathrm{u}_{-1 / 2}, \quad \mathrm{v}_{1 / 2} \rightarrow \frac{1}{2}\left(1-\gamma^{5}\right) \mathrm{v}_{1 / 2}$,

$\mathrm{u}_{1 / 2} \rightarrow \frac{1}{2}\left(1+\gamma^{5}\right) \mathrm{u}_{1 / 2}, \quad \mathrm{v}_{-1 / 2} \rightarrow \frac{1}{2}\left(1+\gamma^{5}\right) \mathrm{v}_{-1 / 2}$.

Thus, anticipating the Standard Model dynamics where only the left-handed chirality of the neutrino and the right-handed chirality of the antineutrino participate, we conclude that one can effectively observe exactly only neutrinos with helicity $-\frac{1}{2}$ and antineutrinos with helicity $\frac{1}{2}$. If the mass of a spacelike neutrino is about or less than $1 \mathrm{eV}$ then this condition is fulfilled already for $q \sim$ tens of $\mathrm{eV}$ so we reproduce the property observed experimentally in the $\mathrm{MeV}$ and $\mathrm{GeV}$ energy range since ever.

On the other hand, if $q \sim \kappa$ then the dependence of the amplitudes $(54,53,56,55)$ on $q$ indicates that both neutrino helicities are present and in consequence one can expect specific predictions to be different from those based on the SM. This regards in particular the electron energy spectrum near the endpoint in $\beta$ decay with the tachyonic neutrino (see Sect. 11). The $q$-dependence of the tachyonic neutrino amplitudes was discussed firstly in Ref. [14] in the context of the reduced model with only one helicity component $\left(-\frac{1}{2}\right.$ for neutrino and $\frac{1}{2}$ for antineutrino, respectively).

\section{Helicity observable}

The helicity operator $\hat{\lambda}$ given in Eq. (22) is realized in the bispinor space as $\hat{\Lambda} \sim u_{\mu} W^{\mu}$, where $W^{\mu}=-\frac{1}{2} \varepsilon^{\mu \nu \tau \sigma} P_{\nu} S_{\tau \sigma}$ is the Pauli-Lubanski pseudo-vector. In the coordinate representation it reads

$\hat{\Lambda}(u) \sim \gamma^{5}\left[\gamma^{\nu}\left(i \partial_{\nu}\right), u_{\mu} \gamma^{\mu}\right]$.

After normalizing in the momentum representation it takes the form

$$
\begin{aligned}
\hat{\Lambda}(k, u) & =\frac{\mp 1}{4 \sqrt{q^{2}+\kappa^{2}}} \gamma^{5}\left[k_{\mu} \gamma^{\mu}, u_{\nu} \gamma^{\nu}\right] \\
& =\mp S\left(L_{u} R_{\mathbf{n}}\right) \frac{1}{2}\left(\begin{array}{cc}
\sigma^{3} & 0 \\
0 & \sigma^{3}
\end{array}\right) \overline{S\left(L_{u} R_{\mathbf{n}}\right)},
\end{aligned}
$$

where the sign - or + is related to the negative (v) or positive (u) frequencies in Eq. (28). In the PF the Weyl representation of $\hat{\Lambda}$ has a simple form

$$
\hat{\Lambda}\left(k, u_{P F}\right)=\mp \frac{1}{2}\left(\begin{array}{cc}
\frac{\mathbf{k}}{|\mathbf{k}|} \cdot \boldsymbol{\sigma} & 0 \\
0 & \frac{\mathbf{k}}{|\mathbf{k}|} \cdot \boldsymbol{\sigma}
\end{array}\right) \text {. }
$$

The action of $\hat{\Lambda}(u, k)$ on the amplitudes can be obtained from $(62,63)$ and $(53,54,55,56)$.

\section{Propagator}

In the standard field theory the propagator is identified with the corresponding Green function (Feynman function) or defined as the vacuum expectation value of time ordered product of field operators. The equivalence of these two approaches is guaranteed by fundamental axioms of relativistic quantum field theory (Wightman axiomatic formulation and LSZ formalism), in particular by the locality and positive definiteness of the Hilbert space, see, e.g., [50,51]. However, one cannot expect commutativity of observables 
separated by space-like intervals in a tachyonic theory. Consequently, the locality axiom cannot be fulfilled in such a case. Therefore, the equivalence of a corresponding Green function with the definition of the propagator as the vacuum expectation value of the time ordered product of quantum fields does not hold. Fortunately, in our case it is possible to redefine the two-point Wightman functions to obtain a propagator equivalent to the Feynman function. The Wightman function $W(x, y)=\langle 0|v(x) \bar{v}(x)| 0\rangle$ calculated with the help of Eqs. $(15,18,19,28)$, has the following, seemingly standard, form

$$
\begin{aligned}
W(x, y) & =\langle 0|v(x) \bar{v}(x)| 0\rangle \\
& =\frac{1}{(2 \pi)^{3}} \int d \mu(k, u) e^{-i k(x-y)} \sum_{\lambda} \mathrm{u}_{\lambda}(k, u) \overline{\mathrm{u}_{\lambda}(k, u)},
\end{aligned}
$$

but using equations from Appendix $\mathrm{C}$ we obtain

$\sum_{\lambda} \mathrm{u}_{\lambda}(k, u) \overline{\mathrm{u}_{\lambda}(k, u)}=\kappa \Pi_{+}\left(\Pi_{1 / 2}-\Pi_{-1 / 2}\right)$,

where the projector $\Pi_{+}$has the form

$\Pi_{+}=\frac{\kappa+\gamma^{5}\left(\gamma^{\mu} k_{\mu}\right)}{2 \kappa}$,

while

$\Pi_{\lambda}=\frac{1}{2}\left(I+\frac{\lambda}{\sqrt{q^{2}+\kappa^{2}}} \gamma^{5}\left[\gamma^{\mu} k_{\mu}, \gamma^{\nu} u_{\nu}\right]\right), \quad \lambda= \pm \frac{1}{2}$

project on the helicity states (compare with Eq. (62)). As it was expected, the above form does not lead to an acceptable $T$-ordered function (it does not lead to a proper Green function). However, if we replace the sum (66) in (65) by the following one

$\sum_{\lambda} \lambda \mathrm{u}_{\lambda}(k, u) \overline{\mathrm{u}_{\lambda}(k, u)}=\frac{\kappa}{2} \Pi_{+}$,

we obtain an acceptable modification of the Wightman function

$$
\begin{aligned}
& W_{\mathcal{T}}(x, y) \\
& =\frac{1}{4(2 \pi)^{3}} \int d \mu(k, u) e^{-i k(x-y)}\left(\kappa+\gamma^{5}\left(\gamma^{\mu} k_{\mu}\right)\right) .
\end{aligned}
$$

The corresponding function $W_{\mathcal{T}}^{T}(y, x)$, related to the Wightman function $\left\langle 0\left|\bar{v}(y)^{T} v(x)^{T}\right| 0\right\rangle$, where the superscript $T$ means transposition, is equal to

$$
\begin{aligned}
& W_{\mathcal{T}}^{T}(y, x) \\
& =\frac{1}{4(2 \pi)^{3}} \int d \mu(k, u) e^{i k(x-y)}\left(\kappa-\gamma^{5}\left(\gamma^{\mu} k_{\mu}\right)\right) .
\end{aligned}
$$

The propagator can be defined analogously to the standard one, i.e.,

$$
S_{\mathcal{T}}(x-y, u)
$$

$$
=\frac{2 i \kappa}{(2 \pi)^{3}}\left(\theta\left(x^{0}-y^{0}\right) W_{\mathcal{T}}(x, y)+\theta\left(y^{0}-x^{0}\right) W_{\mathcal{T}}^{T}(y, x)\right) .
$$

By means of the standard procedure and taking into account the form of the measure $d \mu(k, u)$ given in Eq. (8), integral representation of the theta function (Eq. (A.7)), we obtain from Eqs. $(70,71,72)$

$$
\begin{aligned}
& S_{\mathcal{T}}(x-y, u) \\
& =\frac{1}{2(2 \pi)^{4}} \int d \mu(p, u) E \int_{-\infty}^{\infty} d p^{0} e^{-i p(x-y)} \frac{\kappa+\gamma^{5}\left(\gamma^{\mu} p_{\mu}\right)}{p^{2}+\kappa^{2}+i \eta},
\end{aligned}
$$

where the energy $E=E(q, \theta, u)$ is explicitly given by Eq. (5) while the functional dependence of the measure $d \mu(p, u)=d \mu(q, \theta, u)=\frac{1}{u^{0}}|\mathbf{p}| d \Omega d q \theta(q),|\mathbf{p}|=$ $\omega(q, \theta, u)$ with $\omega$ given by Eq. (6) and the infinitesimal parameter $\eta \sim E \varepsilon$, where $\varepsilon>0$. Notice that, as is evident from Eq. (5), the energy $E$ can take negative values, too. In that case $\eta$ also takes negative values and thus the propagator changes the character from Feynman (causal) to Dyson (anticausal) with respect to the coordinate time $t$. As it follows from Eq. (7), taking the upper limit for the neutrino mass as $\kappa=1 \mathrm{eV}$ and identifying PF with the CMBR frame, this can hold for energies between $0 \mathrm{eV}$ and $-10^{-3} \mathrm{eV}$, i.e., anticausal effects with respect to the coordinate time $t$ are extremely small.

The propagator $S_{\mathcal{T}}(x, u)$ also can be expressed in the form

$$
\begin{aligned}
& S_{\mathcal{T}}(x, u)=\left(\kappa+i \gamma^{5} \gamma^{\mu} \partial_{\mu}\right) \Delta_{T}(x, u), \\
& \quad \text { where } \\
& \Delta_{T}(x, u) \\
& \quad=\frac{1}{2(2 \pi)^{4}} \int d \mu(q, \theta, u) E(q, \theta, u) \int_{-\infty}^{\infty} d p^{0} \frac{e^{-i p x}}{p^{2}+\kappa^{2}+i \eta}
\end{aligned}
$$

is the Green function of the tachyonic Klein-Gordon equation. Recall that the integration measure used by us preserves the structure of the tachyon physical momentum manifold (topologically equivalent to $\mathbb{R}^{3} \backslash B_{\kappa}$ where $B_{\kappa}$ is the open ball of the radius $\kappa$ ). Notice also that for this reason tachyons cannot be sharply localized due to the Heisenberg uncertainty principle.

\section{Discrete symmetries}

It is easy to see that the standard space inversion of a bispinor $v_{P}(x, u)=\gamma^{0} v\left(x^{\pi}, u^{\pi}\right)$,

where $x^{\pi}=\left(x^{0}, \mathbf{x}\right)^{\pi}=\left(x^{0},-\mathbf{x}\right)$ and similarly for other four-vectors, does not preserve the form of the Dirac-like Eq. (48); instead, this equation is form-invariant with respect to the parity transformation defined as

$v_{P}(x, u)=i \gamma^{5} \gamma^{0} v\left(x^{\pi}, u^{\pi}\right)$. 
The Lagrangian

$\mathcal{L}=\overline{v(x, u)}\left(\gamma^{5}\left(\gamma^{\mu} i \partial_{\mu}\right)-\kappa\right) v(x, u)$

related to Eq. (48) is not invariant under the standard inversion (76) and changes sign under the parity transformation (77) so both operations lead to parity nonconservation.

Now, if we introduce the charge conjugation according to the standard procedure (see also Ref. [10]) as

$v_{C}(x, u)=\overline{\mathcal{C}} \overline{(x, u)^{T}}$,

where the unitary matrix $\mathcal{C}$ satisfies the relation $\mathcal{C} \gamma^{\mu T} \mathcal{C}^{T}=$ $\gamma^{\mu}$ in this case (in the Weyl representation $\mathcal{C}=i \gamma^{5} \gamma^{2} \gamma^{0}=$ $\gamma^{3} \gamma^{1}$ ), the Lagrangian $\mathcal{L}$ is also non invariant. Besides the fact that neither the parity nor charge conjugation operations can be realized as symmetries of the tachyonic neutrino Lagrangian even on the free level, their composition is a symmetry. Indeed, it is possible to define a combination of the parity and charge conjugation, $\mathrm{CP}$

$v_{C P}(x, u)=\gamma^{5} \gamma^{0} v_{C}\left(x^{\pi}, u^{\pi}\right)$.

Thus

$$
\begin{aligned}
v_{C P}(x, u)= & \frac{1}{(2 \pi)^{3 / 2}} \int d \mu(k, u) \sum_{\lambda}\left[e^{i k x} \mathrm{v}_{\lambda}(k, u)\right. \\
& \left.\times a_{\lambda}^{\dagger}\left(k^{\pi}, u^{\pi}\right)+e^{-i k x} \mathrm{u}_{\lambda}(k, u) b_{\lambda}\left(k^{\pi}, u^{\pi}\right)\right](81)
\end{aligned}
$$

where we have used the relations $\mathrm{u}_{\lambda}\left(k^{\pi}, u^{\pi}\right)=\gamma^{5} \gamma^{0} \mathrm{u}_{\lambda}(k, u)$ and $\mathrm{v}_{\lambda}\left(k^{\pi}, u^{\pi}\right)=-\gamma^{5} \gamma^{0} \mathbf{v}_{\lambda}(k, u)$ (see Appendix C).

Next, we connect $v_{C P}(x, u)$ with the action of the unitary operator $\mathcal{Q}$ representing the $\mathrm{CP}$ transformation

$\zeta \nu_{C P}(x, u) \equiv \mathcal{Q} v(x, u) \mathcal{Q}^{\dagger}$,

where $\zeta$ is a phase factor. Consequently, by means of Eq. (81)

$$
\begin{aligned}
& \mathcal{Q} a_{\lambda}(k, u) \mathcal{Q}^{\dagger}=\zeta b_{\lambda}\left(k^{\pi}, u^{\pi}\right), \\
& \mathcal{Q} b_{\lambda}(k, u) \mathcal{Q}^{\dagger}=\zeta^{*} a_{\lambda}\left(k^{\pi}, u^{\pi}\right)
\end{aligned}
$$

and the remaining relations are obtained by the Hermitian conjugation and exchange $(k, u)$ with $\left(k^{\pi}, u^{\pi}\right)$. Concluding, in the case of a tachyonic neutrino one cannot define separate discrete symmetries $\mathrm{C}$ or $\mathrm{P}$; but only the $\mathrm{CP}$ symmetry can be realized.

\section{Interactions}

Let us start with an observation made in the paper by Chodos et al. [10] that the Higgs mechanism can, in principle, lead to a tachyonic fermion as well as to a massive fermion. Indeed, the kinetic part of the massless bispinor field Lagrangian could have two different forms

$$
\bar{\psi}\left(\gamma^{\mu} i \partial_{\mu}\right) \psi \text { or } \bar{\psi} \gamma^{5}\left(\gamma^{\mu} i \partial_{\mu}\right) \psi
$$

leading to equivalent massless Dirac equations

$\left(\gamma^{\mu} i \partial_{\mu}\right) \psi=0$ and $\gamma^{5}\left(\gamma^{\mu} i \partial_{\mu}\right) \psi=0$.

However, mass generation via the Yukawa coupling with the Higgs fields leads to inequivalent free field Lagrangians

$\bar{\psi}\left(\gamma^{\mu} i \partial_{\mu}\right) \psi-m \bar{\psi} \psi$ and $\bar{\psi} \gamma^{5}\left(\gamma^{\mu} i \partial_{\mu}\right) \psi-\kappa \bar{\psi} \psi$,

respectively. The first Lagrangian results in the standard Dirac equation while the second Lagrangian leads to the free field Eq. (48) describing a tachyonic fermion.

On the other hand as was pointed out in [17], before the onset of the spontaneous symmetry breaking process, massless Lagrangians for the neutrino field $v$ and the charged lepton field $l$ had the form $\mathcal{L}_{0}^{I}$ in the standard case (leading to two Dirac leptons, $l$ and $v$ ) and $\mathcal{L}_{0}^{I I}$ in the mixed case (leading to a Dirac lepton and a tachyonic neutrino)

$$
\begin{aligned}
\mathcal{L}_{0}^{I} & =\bar{l}\left(\gamma^{\mu} i \partial_{\mu}\right) l+\bar{v}\left(\gamma^{\mu} i \partial_{\mu}\right) \nu, \\
\mathcal{L}_{0}^{I I} & =\bar{l}\left(\gamma^{\mu} i \partial_{\mu}\right) l+\bar{v} \gamma^{5}\left(\gamma^{\mu} i \partial_{\mu}\right) \nu .
\end{aligned}
$$

Now, in the chiral representation of the fields $v$ and $l$, Lagrangian $\mathcal{L}_{0}^{I}$ is invariant under $\left(\mathrm{SU}(2)_{L} \times \mathrm{U}(1)_{L}\right) \times$ $\left(\mathrm{SU}(2)_{R} \times \mathrm{U}(1)_{R}\right)$ transformations of the chiral left doublet $L$ and the right doublet $R$. On the other hand, Lagrangian $\mathcal{L}_{0}^{I I}$ is invariant under $\left(\mathrm{SU}(2)_{L} \times \mathrm{U}(1)_{L}\right)$ transformation of the left doublet $L$ and under $\left(\mathrm{U}(1)_{I_{3} R} \times \mathrm{U}(1)_{R}\right)$ subgroup of the right $\left(\mathrm{SU}(2)_{R} \times \mathrm{U}(1)_{R}\right)$ group. Therefore, $\mathcal{L}_{0}^{I I}$ admits only a doublet $L$ and two singlets, $v_{R}$ and $l_{R}$, exactly as one needs for a formulation of the electroweak sector of the Standard Model. Concluding, the mixed Lagrangian (89) fixes exactly the weak group and its representation without additional requirements.

By means of the standard procedure of gauging and the spontaneous symmetry breaking up to the electromagnetic gauge group $\bigcup(1)_{Q E D}$ we obtain a Lagrangian which differs from the SM Lagrangian by the neutrino kinetic term only. In the unitary gauge the leptonic part of this Lagrangian takes the usual form

$$
\begin{aligned}
\mathcal{L}_{\text {lepton }}= & \bar{l}\left(\gamma^{\mu} i \partial_{\mu}-m_{l}\right) l+\bar{v}\left(\gamma^{5} \gamma^{\mu} i \partial_{\mu}-\kappa\right) v \\
& +\frac{g}{2 \sqrt{2}}\left(W_{\mu}^{-} j_{+}^{\mu}+W_{\mu}^{+} j_{-}^{\mu}\right)+\frac{g}{2 \cos \theta_{W}} Z_{\mu}\left(j_{l}^{\mu}+j_{v L}^{\mu}\right) \\
& -e A_{\mu} j_{e}^{\mu}-\frac{m_{l}}{v} \bar{l} H l-\frac{\kappa}{v} \bar{v} H v,
\end{aligned}
$$

where $g=e / \sin \theta_{W}, v^{2}=1 /\left(\sqrt{2} G_{F}\right), e$ is the electric charge, $G_{F}-$ the Fermi constant, $\theta_{W}$ - the Weinberg angle, $m_{l}-$ mass of the lepton $l$. Here, $H$ denotes the Higgs field, $W_{\mu}^{ \pm}$and $Z_{\mu}$ are the charged and neutral weak bosons fields, respectively, and $A_{\mu}$ the electromagnetic fourpotential. The electroweak currents take also the standard form $j_{+}^{\mu}=\bar{l} \gamma^{\mu}\left(1-\gamma^{5}\right) \nu, j_{-}^{\mu}=\bar{v} \gamma^{\mu}\left(1-\gamma^{5}\right) l, j_{e}^{\mu}=\bar{l} \gamma^{\mu} l$, $j_{l}^{\mu}=\bar{l} \gamma^{\mu}\left(g_{V}-g_{A} \gamma^{5}\right) l, j_{v L}^{\mu}=\frac{1}{2} \bar{v} \gamma^{\mu}\left(1-\gamma^{5}\right) \nu, j_{v R}^{\mu}=$ $\frac{1}{2} \bar{v} \gamma^{\mu}\left(1+\gamma^{5}\right) \nu$, where $g_{V}=2 \sin ^{2} \theta_{W}-\frac{1}{2}, g_{A}=-\frac{1}{2}$. 
Taking into account the form of the neutrino amplitudes discussed in Sect. 6, Lagrangian (90), at least in the tree approximation, leads to the SM results for energies significantly higher than the neutrino mass $\kappa$ (as well as to an agreement with the model reduced to the one helicity component discussed in $[14,16])$. However, for energies close to the tachyonic neutrino mass $\kappa$, where both the left and right handed chiral components are present, one could expect new effects.

\section{Beta decay of ${ }^{3} \mathrm{H}$}

Tritium decay with a space-like neutrino was discussed by us within the framework of the reduced model [16]; the corresponding differential decay rate (electron energy spectrum) shows an anomalous energy dependence close to the endpoint. Earlier calculations of the Kurie plot [10] also indicated differences compared to the prediction for a massive neutrino. Below we derive the corresponding expressions for the complete model, i.e., with the lepton interaction Lagrangian given by (90), taking into account the contributions of all two chiral and helicity components of the tachyonic neutrino field.

The amplitude squared of this process, $|M|^{2}$, is given at the tree level by the formula

$$
\begin{aligned}
|M|^{2} \sim & G_{F}^{2} \operatorname{Tr}\left[\left(l \gamma+m_{e}\right) \gamma^{\mu}\left(1-\gamma^{5}\right)\left(\gamma^{5} k \gamma+\kappa\right)\right. \\
& \left.\times \gamma^{v}\left(1-\gamma^{5}\right)\right] \operatorname{Tr}\left[\left(p \gamma+m_{P}\right) \gamma_{\mu}\left(I-g_{A} \gamma^{5}\right)\right. \\
& \left.\times\left(r \gamma+m_{N}\right) \gamma_{v}\left(I-g_{A} \gamma^{5}\right)\right],
\end{aligned}
$$

i.e.,

$$
\begin{aligned}
& |M|^{2} \sim 2 g_{A}[(l r)(k p)-(l p)(k r)]-\left(1+g_{A}^{2}\right)[(l p)(k r) \\
& \quad+(l r)(k p)]+\left(1-g_{A}^{2}\right) m_{N} m_{P}(k l),
\end{aligned}
$$

where $m_{N}, m_{P}, m_{e}, \kappa$ and $r, p, l, k$ are the masses and fourmomenta of tritium ${ }^{3} \mathrm{H}$, helium ${ }^{3} \mathrm{He}$, electron and tachyonic anti-neutrino, respectively. By means of the above formulas one can obtain the differential decay rate in terms of the outgoing electron energy, $E$, in the form

$\frac{d \Gamma}{d E}=\frac{1}{128 \pi^{3} m_{N}} \int_{\max \left\{0, k_{-}\right\}}^{k_{+}}|M|^{2} d q$,

where, the limiting values of the neutrino energy following from the tachyonic kinematics in the preferred frame are

$$
\begin{aligned}
k_{ \pm}= & {\left[\left(m_{N}-l_{0}\right)\left(\Delta m^{2}-2 l^{0} m_{N}\right) \pm\left\{\left(l^{0^{2}}-m_{e}^{2}\right)\right.\right.} \\
& \left.\left.\times\left[\left(\Delta m^{2}-2 l^{0} m_{N}\right)^{2}+4 \kappa^{2}\left(m_{e}^{2}+m_{N}^{2}-2 l^{0} m_{N}\right)\right]\right\}\right] \\
& \times\left[2\left(m_{e}^{2}+m_{N}^{2}-2 l^{0} m_{N}\right)\right]^{-1},
\end{aligned}
$$

where $l^{0}=E+m_{e}$ and $\Delta m^{2}=\left(m_{N}^{2}-m_{P}^{2}\right)+\left(m_{e}^{2}-\kappa^{2}\right)$.

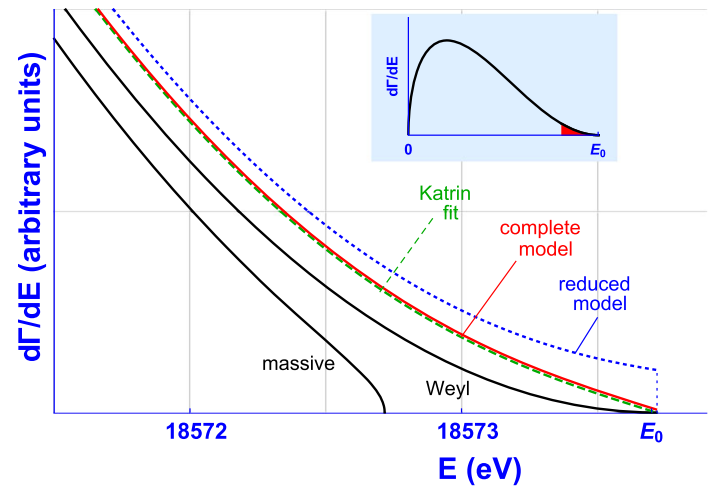

Fig. 1 Theoretical electron energy spectrum (differential decay rate) near the endpoint in tritium decay at rest; $E_{0}=18573.7 \mathrm{eV}$ is the endpoint energy fitted by KATRIN. The curves demonstrate the cases for: massive neutrino with $m_{v}=1 \mathrm{eV}$ (the kinematical limit in the decay with a massive neutrino is $E_{0}-m_{\nu}$ ); massless (Weyl) neutrino; massive neutrino with inverted sign of $m_{v}^{2}$ illustrating the outcome of the KATRIN fit to their data (dashed line); complete model (solid line) and the reduced model (dotted line) for a tachyonic neutrino with $\kappa=1 \mathrm{eV}$. The curves for the complete model and the KATRIN fit overlap in reality but have been infinitesimally shifted for the purpose of illustration. Inset: figurative visualization of the range covered on the main plot w.r.t. the full electron energy spectrum within bounds $\left[0, E_{0}\right]$

The above differential decay rate $d \Gamma / d E$, as a function of the electron kinetic energy, is presented in Fig. 1 together with the corresponding curve predicted in the reduced model [16]. We also show curves for a massive neutrino and massless Weyl neutrino. The predictions of the complete and the reduced models are slightly different near the endpoint which allows (under a working hypothesis that the neutrino is a tachyonic fermion) to test both possibilities experimentally.

Recently the KATRIN Collaboration delivered the most precise measurement of the neutrino mass squared in tritium decay: $m_{v_{e}}^{2}=-1.0_{-1.1}^{+0.9} \mathrm{eV}^{2}[4,5]$. The prediction of the complete model with $\kappa^{2}=1 \mathrm{eV}^{2}$ and the recent KATRIN fit representing the above value are indistinguishable. The KATRIN result, despite its yet insufficient statistical significance, is a subsequent one in a row yielding negative central values for the fitted neutrino mass squared, following earlier measurements at Mainz [6,8] and Troitsk [7,9].

\section{Conclusions}

We have presented for the first time a fully consistent formalism of quantization of space-like fermions with helicity $\pm \frac{1}{2}$. In our formalism all common concerns have been resolved and explained. Specifically, (i) negative energy problem is solved and no imaginary energies appear, (ii) causality paradoxes do not appear, (iii) the proper quantization procedure has been described in detail (Hilbert space with suitable Poincare properties indicated), (iv) interactions are unitary 
and theory is perturbatively renormalizable (anomaly free), (v) since the presented description is Lorentz covariant, all properties apply to arbitrary reference frames (and not only the preferred frame). In order to achieve that and to avoid causal problems with tachyons, existence of a preferred frame must be assumed; it is natural to suppose that the PF coincides with the Cosmic Neutrino Background frame. We again indicate that the preferred frame, if exists in nature, would be uniquely a part of the tachyonic sector and not that of conventional physics that describes light and slower than light (massive) objects. For those the preferred frame is but an ordinary inertial frame respecting the principles of the Einsteinian relativity. The preferred frame cannot be discovered with the use of light or massive particles owing to the intrinsic properties of the Einsteinian relativity, in particular the corresponding clock synchronisation procedure. We determined the unitary realizations of the inhomogenous Lorentz group by means of the Wigner-Mackey induction procedure and constructed the corresponding Fock space. In the preferred frame scenario, the irreducible unitary realizations of the Lorentz group for tachyons are labeled by particle helicity (not by spin). The ultimate, manifestly Lorentz covariant formalism was developed by way of constructing the field amplitudes according to the Weinberg method. Since our theoretical findings regarding the space-like helicity $\pm \frac{1}{2}$ fermions suggest connotations with neutrinos, we show that indeed the following facts and observations from the field of neutrino physics can be interpreted within the presented approach at the level of fundamental properties. According to our formalism, only neutrinos with helicity $-\frac{1}{2}$ and antineutrinos with helicity $+\frac{1}{2}$ can be effectively observed at asymptotic energies (significantly exceeding the neutrino mass, $\kappa$, amounting to or a fraction of a single eV) which is a well established knowledge based on the lack of experimental evidence for the complementary cases at $\mathrm{MeV}$-and higher energies. It should be stressed that in our formalism this fact would be a consequence solely of the "tachyoneity" of the neutrino, i.e., its intrinsic property at the free level and not exclusively due to the observed character of weak interactions. Likewise would be the chiral asymmetry of weak interactions, thus far introduced in an explicit way to the structure of the neutrino current. These inherent features of space-like fermions follow directly from the energy dependence of the amplitudes for asymptotic energies $q \gg \kappa$, described by Eqs. $(57,58,59,60)$. This allows to explain the mysterious chiral properties of the neutrinos as well as occurrence of only $-\frac{1}{2}$ helicity neutrino and its conjugate in nature, both age-old experimental observations. In addition, we have also shown that neither $\mathrm{C}$ nor $\mathrm{P}$ symmetry holds for space-like helicity $\pm \frac{1}{2}$ fermions at the free level but rather the combined $\mathrm{CP}$ symmetry is conserved. The nature of this property is intrinsic if neutrinos are tachyons and not arising due to their interactions.
It is useful to discuss shortly similarities and differences between our construction of the tachyonic propagator and the approach given in the recent paper by Jentschura and Wundt [22]. Their construction assumes an indefinite-norm Hilbert space equipped with unconventional canonical anticommutation relations (CACR) dependent on neutrino chiralities. We adopt the usual CACR, so from the beginning we deal with a positive-norm Hilbert space. Next, the authors of [22] use a formalism analogous to the Gupta-Bleuler approach in the quantum electrodynamics. The unobservable neutrino/antineutrino polarizations are related to the states with the negative norm and thus are unphysical. In our approach these polarizations are physical, however rapidly decreasing with growing energy which leads to the same effect. In contrast to our work, the authors of [22] do not assume a preferred frame scenario. Their propagator is constructed with a modification of the sum of products of amplitudes similar to ours (Eq. (69)) but with relations to chirality while in our approach a similar role is played by helicity. However, there is an essential difference between both formalisms. Namely, as indicated in Sect. 8, the integration measure used by us preserves the structure of the tachyon physical momentum manifold. In [22] the momentum manifold includes also unphysical momenta from $B_{\kappa}$, i.e., is identified with the whole space $\mathbb{R}^{3}$. This forced appearance of resonant states as discussed in [22]. For the above reasons the propagators in our approach and in [22] are different, irrespectively of their superficial similarity.

As was mentioned in Sect. 10, the mixed free field Lagrangian (89) yet before of the spontaneous symmetry breaking admits only the Weinberg-Salam weak group and its representation without additional requirements. After gauging and the electroweak symmetry breaking in the lepton sector one obtains finally the Lagrangian (90), identical to the SM Lagrangian except of the neutrino kinetic term. The resulting model is anomaly free and perturbatively renormalizable.

Tachyonic neutrinos also can oscillate according to the same pattern as massive neutrinos; oscillations in the present formulation do not distinguish between the massive and tachyonic forms of the dispersion relation [19].

In the low energy neutrino limit, the negative value of the neutrino mass squared, determined in several recent tritium decay experiments, makes an interesting observation, though not yet conclusive but pointing to the hypothesis of a spacelike nature of the neutrino. As we have shown in Fig. 1, the conventional fit, recently confirmed by KATRIN, coincides with the prediction of our complete model under the assumption that the tachyonic neutrino mass amounts to $\kappa=1 \mathrm{eV}$.

On the other hand, tachyonic neutrinos are the Dirac particles in our approach. Thus one way of falsifying the hypothesis that neutrinos are tachyons would be to observe the neutrinoless double beta decay indicating that neutrinos were the 
Majorana particles. This process has not been discovered to date despite several decades of experimenting.

Data Availability Statement This manuscript has no associated data or the data will not be deposited. [Authors' comment: This is a theoretical paper and does not require the use of any data other then these contained in standard tables and cited references.]

Open Access This article is licensed under a Creative Commons Attribution 4.0 International License, which permits use, sharing, adaptation, distribution and reproduction in any medium or format, as long as you give appropriate credit to the original author(s) and the source, provide a link to the Creative Commons licence, and indicate if changes were made. The images or other third party material in this article are included in the article's Creative Commons licence, unless indicated otherwise in a credit line to the material. If material is not included in the article's Creative Commons licence and your intended use is not permitted by statutory regulation or exceeds the permitted use, you will need to obtain permission directly from the copyright holder. To view a copy of this licence, visit http://creativecomm ons.org/licenses/by/4.0/.

Funded by SCOAP ${ }^{3}$.

\section{Appendix A: Invariant measure, the spherical Dirac delta and completeness relation}

The Lorentz invariant measure taking into account existence of the preferred frame has the following form

$$
\begin{aligned}
d \mu(k, u) & =d^{4} k \delta\left(k^{2}+\kappa^{2}\right) \Theta(u k) \\
& =d k^{0}|\mathbf{k}|^{2} d|\mathbf{k}| d \Omega \Theta(u k) \delta\left(|\mathbf{k}|^{2}-\omega^{2}\right) \\
& =d k^{0}|\mathbf{k}|^{2} d|\mathbf{k}| d \Omega \Theta(u k) \frac{\delta(|\mathbf{k}|-\omega)}{2 \omega} \\
& =\frac{\omega(q, u, \theta)}{2 u^{0}} \Theta(q) d q d \Omega .
\end{aligned}
$$

Here $\theta$ is the angle between $\mathbf{u}$ and $\mathbf{k}, \omega(q, u, \theta)$ is given in Eq. (6). Furthermore $\Theta$ is the Heaviside Theta and

$$
\begin{aligned}
d^{4} k & =d k^{0} \wedge d^{3} \mathbf{k} \\
& =\frac{1}{u^{0}} d(q+\mathbf{u} \cdot \mathbf{k}) \wedge d^{3} \mathbf{k} \\
& =\frac{1}{u^{0}} d q \wedge d^{3} \mathbf{k} \\
& =\frac{1}{u^{0}} d q d^{3} \mathbf{k} .
\end{aligned}
$$

The spherical Dirac delta is the angular part of the three dimensional Dirac delta. It is defined as follows: Let $\mathbf{n}(\theta, \varphi)$ denote a unit vector depending on the spherical coordinates (angles) $\theta$ and $\varphi$. Then the spherical Dirac delta is defined by the formula

$$
\int_{S_{2}} d \Omega \delta\left(\mathbf{n}-\mathbf{n}_{0}\right) f(\mathbf{n})=f\left(\mathbf{n}_{0}\right),
$$

where $\mathbf{n}_{0}=\mathbf{n}\left(\theta_{0}, \varphi_{0}\right)$ and $d \Omega$ is the solid angle differential. In terms of the angles $\theta$ and $\varphi d \Omega=\sin \theta d \theta d \varphi$ and $\delta(\mathbf{n}-$ $\left.\mathbf{n}_{0}\right)=\frac{\delta\left(\theta-\theta_{0}\right) \delta\left(\varphi-\varphi_{0}\right)}{\sin \theta}$.
Below we prove explicitly the completeness relation (14). Since neutrino and antineutrino spaces are mutually orthogonal, it is enough to prove this relation in one of those spaces, say neutrino space of states. Therefore, we are to prove that for any vector

$$
|\psi, u\rangle=\sum_{\lambda^{\prime}} \int d \mu(p, u) \psi\left(p, u, \lambda^{\prime}\right)\left|p, u, \lambda^{\prime}\right\rangle
$$

from one-particle neutrino space it holds

$$
\sum_{\lambda} \int d \mu(k, u)(|k, u, \lambda\rangle\langle k, u, \lambda|)|\psi, u\rangle=|\psi, u\rangle .
$$

Indeed, we have

$$
\begin{aligned}
(|k, u, \lambda\rangle\langle k, u, \lambda|)|\psi, u\rangle \\
=\sum_{\lambda^{\prime}} \int d \mu(p, u) \psi\left(p, u, \lambda^{\prime}\right)\left\langle k, u, \lambda \mid p, u, \lambda^{\prime}\right\rangle|k, u, \lambda\rangle \\
=\sum_{\lambda^{\prime}} \int d q_{p} d \Omega\left(\theta_{p}, \varphi_{p}\right) \frac{\omega\left(q_{p}, u, \theta_{p}\right)}{2 u^{0}} \Theta\left(q_{p}\right) \\
\quad \times \frac{2 u^{0}}{\omega\left(q_{k}, u, \theta_{k}\right)} \delta_{\lambda \lambda^{\prime}} \delta\left(q_{k}-q_{p}\right) \delta(\hat{\mathbf{k}}-\hat{\mathbf{p}}) \\
\quad \times \psi\left(p, u, \lambda^{\prime}\right)|k, u, \lambda\rangle \\
=\psi(k, u, \lambda)|k, u, \lambda\rangle
\end{aligned}
$$

where we used Eqs. (8, 13, A.3). This last equation immediately implies Eq. (A.5). When calculating the propagator we use the following representation of the theta function

$\theta(t)=\frac{1}{2 \pi i} \lim _{\varepsilon \rightarrow 0^{+}} \int_{-\infty}^{\infty} d \chi \frac{e^{i t \chi}}{\chi-i \varepsilon}$.

\section{Appendix B: Rotations, boosts etc.}

The rotation $R_{\mathbf{n}}$ reduced to the space sector has the form

$$
R_{\mathbf{n}}=\left(\begin{array}{ccc}
1-\frac{\left(n^{1}\right)^{2}}{1+n^{3}} & -\frac{n^{1} n^{2}}{1+n^{3}} & n^{1} \\
-\frac{n^{1} n^{2}}{1+n^{3}} & 1-\frac{\left(n^{2}\right)^{2}}{1+n^{3}} n^{2} \\
-n^{1} & -n^{2} & n^{3}
\end{array}\right)
$$

while its spinor counterpart $\mathbb{U}_{\mathbf{n}}$

$\mathbb{U}_{\mathbf{n}}=\frac{1}{\sqrt{2\left(1+n^{3}\right)}}\left(\begin{array}{cc}1+n^{3} & -n^{1}+i n^{2} \\ n^{1}+i n^{2} & 1+n^{3}\end{array}\right)$.

Here the unit vector $\mathbf{n}(k, u)$ is given by Eq. (10). Boost and its spinor representative have the form

$L_{u}=\left(\begin{array}{cc}u^{0} & \mathbf{u}^{T} \\ \mathbf{u} & I+\frac{\mathbf{u} \otimes \mathbf{u}^{T}}{1+u^{0}}\end{array}\right)$, 
$\mathbb{L}_{u}=\frac{1}{\sqrt{2\left(1+u^{0}\right)}}\left(\left(1+u^{0}\right) I-\mathbf{u} \cdot \boldsymbol{\sigma}\right)$.

One can also show that the following relations hold

$$
\begin{aligned}
\mathbb{L}_{u}^{\dagger} & =\mathbb{L}_{u}, \quad \mathbb{L}_{u^{\pi}}=\mathbb{L}_{u}^{-1} \\
S\left(L_{u} R_{\mathbf{n}}\right) & =\left(\begin{array}{cc}
\mathbb{L}_{u} \mathbb{U}_{\mathbf{n}} & 0 \\
0 & \mathbb{L}_{u}^{-1} \mathbb{U}_{\mathbf{n}}
\end{array}\right), \\
S^{-1}\left(L_{u} R_{\mathbf{n}}\right) & =\frac{\overline{S\left(L_{u} R_{\mathbf{n}}\right)} .}{}
\end{aligned}
$$

Let $k \sigma=k^{0} I-\mathbf{k} \cdot \boldsymbol{\sigma}, k^{\pi} \sigma=k^{0} I+\mathbf{k} \cdot \boldsymbol{\sigma}$, and $\tilde{k} \sigma=$ $q I-\sqrt{q^{2}+\kappa^{2}} \sigma^{3}$. Then

$$
\begin{aligned}
k \sigma & =\mathbb{L}_{u} \mathbb{U}_{\mathbf{n}}(\tilde{k} \sigma) \mathbb{U}_{\mathbf{n}}^{\dagger} \mathbb{L}_{u} \\
& =\mathbb{L}_{u}\left(q I-\sqrt{q^{2}+\kappa^{2}} \mathbf{n} \cdot \boldsymbol{\sigma}\right) \mathbb{L}_{u}, \\
k^{\pi} \sigma & =\mathbb{L}_{u}^{-1} \mathbb{U}_{\mathbf{n}}\left(\tilde{k}^{\pi} \sigma\right) \mathbb{U}_{\mathbf{n}}^{\dagger} \mathbb{L}_{u}^{-1} \\
& =\mathbb{L}_{u}^{-1}\left(q I+\sqrt{q^{2}+\kappa^{2}} \mathbf{n} \cdot \boldsymbol{\sigma}\right) \mathbb{L}_{u}^{-1},
\end{aligned}
$$

and

$\mathbb{L}_{u} \sigma^{0} \mathbb{L}_{u}=\mathbb{L}_{u}^{2}=u^{0} I-\mathbf{u} \cdot \boldsymbol{\sigma}$,

where

$\mathbf{n} \cdot \boldsymbol{\sigma}=\left(\begin{array}{cc}n^{3} & n^{1}-i n^{2} \\ n^{1}+i n^{2} & -n^{3}\end{array}\right)=\mathbb{U}_{\mathbf{n}} \sigma^{3} \mathbb{U}_{\mathbf{n}}^{\dagger}$

and

$\mathbf{n}=\mathbf{n}(k, u)=\frac{1}{\sqrt{q^{2}+\kappa^{2}}}\left(\mathbf{k}-\frac{q+k^{0}}{1+u^{0}} \mathbf{u}\right)$.

Moreover

$\sigma^{2} \mathbb{U}_{\mathbf{n}}^{*} \sigma^{2}=\mathbb{U}_{\mathbf{n}}, \quad \sigma^{2} \mathbb{L}_{u}^{T} \sigma^{2}=\mathbb{L}_{u}^{-1}$.

\section{Appendix C: Relations between amplitudes: Space- inverted amplitudes, scalar products and polarization operators}

The amplitudes given in $(53,54,55,56)$ satisfy a number of relations listed in this Appendix. Taking into account the fact that in the case of the space inverted pair $\left(k^{\pi}, u^{\pi}\right)$, we have $\left(k^{\pi}, u^{\pi}\right)=\mathbb{L}_{u^{\pi}} R_{\mathbf{n}}\left(\tilde{k}^{\pi}, u_{P F}\right)$, where $\tilde{k}^{\pi}=$ $\left(q, 0,0,-\sqrt{q^{2}+\kappa^{2}}\right)$, we can obtain space inverted amplitudes. To do this, $\tilde{k}$ should be replaced by $\tilde{k}^{\pi}$ and $\mathbb{L}_{u}$ by $\mathbb{L}_{u^{\pi}}$ in the formulas $(36,37)$. Notice, that the following relations hold

$$
\begin{aligned}
\mathrm{u}_{\lambda}\left(k^{\pi}, u^{\pi}\right) & =\gamma^{5} \gamma^{0} \mathbf{u}_{\lambda}(k, u), \\
\mathrm{v}_{\lambda}\left(k^{\pi}, u^{\pi}\right) & =-\gamma^{5} \gamma^{0} \mathbf{v}_{\lambda}(k, u)=-\gamma^{0} \mathbf{u}_{-\lambda}(k, u), \\
\mathrm{u}_{\lambda}(k, u) & =-\gamma^{5} \mathbf{v}_{-\lambda}(k, u) .
\end{aligned}
$$

The scalar products of the amplitudes take the form

$$
\begin{aligned}
& \overline{\mathrm{u}_{\lambda}(k, u)} \mathrm{u}_{\sigma}(k, u)=2 \lambda \delta_{\lambda \sigma}, \\
& \overline{\mathrm{v}_{\lambda}(k, u)} \mathrm{v}_{\sigma}(k, u)=2 \lambda \delta_{\lambda \sigma}, \\
& \overline{\mathrm{u}_{\lambda}(k, u)} \mathrm{v}_{\sigma}(k, u)=\overline{\mathrm{v}_{\lambda}(k, u)} \mathrm{u}_{\sigma}(k, u)=0, \\
& \overline{\mathrm{u}_{\lambda}(k, u)} \gamma^{5} \gamma^{0} \mathbf{u}_{\sigma}(k, u)=-2 \lambda k^{0} \delta_{\lambda \sigma}, \\
& \overline{\mathrm{v}_{\lambda}(k, u)} \gamma^{5} \gamma^{0} \mathbf{v}_{\sigma}(k, u)=2 \lambda k^{0} \delta_{\lambda \sigma}, \\
& \overline{\mathrm{u}_{\lambda}(k, u)} \gamma^{5} \gamma^{0} \mathbf{v}_{\sigma}\left(k^{\pi}, u^{\pi}\right)=0, \\
& \overline{\mathrm{v}_{\lambda}(k, u)} \gamma^{5} \gamma^{0} \mathbf{u}_{\sigma}\left(k^{\pi}, u^{\pi}\right)=0 . \\
& \left(\kappa+\gamma^{5}\left(\gamma^{\mu} k_{\mu}\right)\right)=\sum_{\lambda} 2 \lambda \mathrm{u}_{\lambda}(k, u) \overline{\mathrm{u}_{\lambda}(k, u)} \\
& \quad=S\left(L_{u} R_{\mathbf{n}}\right)\left(\kappa+\gamma^{5}\left(\gamma^{\mu} \tilde{k}_{\mu}\right)\right) S\left(L_{u} R_{\mathbf{n}}\right)^{-1}, \\
& \left(\kappa-\gamma^{5}\left(\gamma^{\mu} k_{\mu}\right)\right)=\sum_{\lambda} 2 \lambda \mathrm{v}_{\lambda}(k, u) \overline{\mathrm{v}_{\lambda}(k, u)} \\
& =S\left(L_{u} R_{\mathbf{n}}\right)\left(\kappa-\gamma^{5}\left(\gamma^{\mu} \tilde{k}_{\mu}\right)\right) S\left(L_{u} R_{\mathbf{n}}\right)^{-1},
\end{aligned}
$$

Defining projectors

$\Pi_{+}=\frac{1}{2 \kappa}\left(\kappa+\gamma^{5}\left(\gamma^{\mu} k_{\mu}\right)\right), \quad \Pi_{-}=\frac{1}{2 \kappa}\left(\kappa-\gamma^{5}\left(\gamma^{\mu} k_{\mu}\right)\right)$

we obtain

$\Pi_{+}+\Pi_{-}=I$,

$\Pi_{+} \mathrm{u}_{\lambda}(k, u)=\mathrm{u}_{\lambda}(k, u), \quad \Pi_{+} \mathrm{v}_{\lambda}(k, u)=0$,

$\Pi_{-} \mathrm{v}_{\lambda}(k, u)=\mathrm{v}_{\lambda}(k, u), \quad \Pi_{-} \mathrm{u}_{\lambda}(k, u)=0$.

Projectors on helicity states have the following form

$\Pi_{\lambda}=\frac{1}{2}\left(I+\frac{\lambda}{\sqrt{q^{2}+\kappa^{2}}} \gamma^{5}\left[\gamma^{\mu} k_{\mu}, \gamma^{\nu} u_{\nu}\right]\right), \quad \lambda= \pm \frac{1}{2}$

and the following holds

$\left[\Pi_{ \pm}, \Pi_{\lambda}\right]=0$.

Moreover, we use the relations

$$
\begin{aligned}
\mathrm{u}_{\lambda}(k, u) \overline{\mathrm{u}_{\lambda}(k, u)} & =2 \kappa \lambda \Pi_{+} \Pi_{\lambda}, \\
\mathrm{v}_{\lambda}(k, u) \overline{\mathrm{v}_{\lambda}(k, u)} & =2 \kappa \lambda \Pi_{-} \Pi_{-\lambda}, \\
\sum_{\lambda} \mathrm{u}_{\lambda}(k, u) \overline{\mathrm{u}_{\lambda}(k, u)} & =\kappa \Pi_{+}\left(\Pi_{1 / 2}-\Pi_{-1 / 2}\right), \\
\sum_{\lambda} \mathrm{v}_{\lambda}(k, u) \overline{\mathrm{v}_{\lambda}(k, u)} & =-\kappa \Pi_{-}\left(\Pi_{1 / 2}-\Pi_{-1 / 2}\right), \\
\sum_{\lambda} \lambda \mathrm{u}_{\lambda}(k, u) \overline{\mathrm{u}_{\lambda}(k, u)} & =\frac{\kappa}{2} \Pi_{+}, \\
\sum_{\lambda} \lambda \mathrm{v}_{\lambda}(k, u) \overline{\mathrm{v}_{\lambda}(k, u)} & =\frac{\kappa}{2} \Pi_{-}
\end{aligned}
$$




\section{References}

1. R.N. Mohapatra, G. Senjanović, Phys. Rev. Lett. 44, 912 (1980)

2. M. Magg, C. Wetterich, Phys. Lett. B 94, 61 (1980)

3. J. Schechter, J.W.F. Valle, Phys. Rev. D 22, 2227 (1980)

4. M. Aker et al., Phys. Rev. Lett. 123, 221802 (2019)

5. M. Aker et al., First operation of the KATRIN experiment with tritium (2019). arXiv:1909.06069

6. C. Weinheimer, B. Degenddag, A. Bleile, J. Bonn, L. Bornschein, O. Kazachenko, A. Kovalik, E. Otten, Phys. Lett. B 460(1), 219 (1999)

7. V. Lobashev et al., Phys. Lett. B 460(1), 227 (1999)

8. C. Kraus et al., Eur. Phys. J. C 40, 447 (2005)

9. V.N. Aseev et al., Phys. Rev. D 84, 112003 (2011)

10. A. Chodos, A.I. Hauser, V.A. Kostelecký, Phys. Lett. B 150(6), 431 (1985)

11. E. Giannetto, G. Maccarrone, R. Mignani, E. Recami, Phys. Lett. B 178(1), 115 (1986)

12. K. Kamoi, S. Kamefuchi, Prog. Theor. Phys. 45(5), 1646 (1971)

13. N. Nakanishi, Progr. Theor. Phys. Suppl. 51, 1 (1972)

14. J. Rembieliński, Int. J. Mod. Phys. A 12(9), 1677 (1997)

15. D. Baumann, F. Beutler, R. Flauger, D. Green, A. Slosar, M. VargasMagaña, B. Wallisch, C. Yèche, Nat. Phys. 15, 465 (2019)

16. J. Ciborowski, J. Rembieliński, Eur. Phys. J. C 8, 157 (1999)

17. P. Caban, J. Rembieliński, K.A. Smoliński, Concepts Phys. III, 79 (2003). arXiv:hep-ph/9707391

18. M.J. Radzikowski, in CPT and Lorentz Symmetry, ed. by V.A. Kostelecký (World Scientific, 2010), pp. 224-228

19. P. Caban, J. Rembieliński, K.A. Smoliński, Z. Walczak, Found. Phys. Lett. 19(6), 619 (2006)

20. A. Chodos, V.A. Kostelecký, R. Potting, E. Gates, Mod. Phys. Lett. A 07(06), 467 (1992)

21. R. Ehrlich, Astropart. Phys. 66, 11 (2015)

22. U.D. Jentschura, B.J. Wundt, Eur. Phys. J. C 72, 1894 (2012)

23. U.D. Jentschura, I. Nándori, R. Ehrlich, J. Phys. G Nucl. Part. Phys. 44(10), 105201 (2017)

24. G. Somogyi, I. Nándori, U.D. Jentschura, Phys. Rev. D 100, 035036 (2019)

25. P.A.M. Dirac, Nature 168, 906 (1951)

26. P.A.M. Dirac, Proc. R. Soc. Lond. A 209, 291 (1951)

27. P.C.W. Davies, J.R. Brown (eds.), The Ghost in the Atom (Cambridge University Press, Cambridge, 1986)
28. D. Bohm, Phys. Rev. 85, 166 (1952)

29. J.S. Bell, in Quantum Gravity 2. ed. by C. Isham, R. Penrose, D. Sciama (Oxford University Press, New York, 1981), pp. 611-637

30. N. Gisin, in Quantum Theory: A Two-Time Success Story, ed. by D.C. Struppa, J.M. Tollaksen (Springer, 2014), pp. 185-204

31. H. Zbinden, J. Brendel, N. Gisin, W. Tittel, Phys. Rev. A 63, 022111 (2001)

32. P. Caban, J. Rembieliński, Phys. Rev. A 59, 4187 (1999)

33. J. Rembieliński, J. Ciborowski, Phys. Rev. A 97, 062106 (2018)

34. J. Rembieliński, K.A. Smoliński, Phys. Rev. A 66, 052114 (2002)

35. K. Kowalski, J. Rembieliński, K.A. Smoliński, Phys. Rev. D 76, 045018 (2007)

36. D. Colladay, V.A. Kostelecký, Phys. Rev. D 55, 6760 (1997)

37. D. Colladay, V.A. Kostelecký, Phys. Rev. D 58, 116002 (1998)

38. S. Coleman, S.L. Glashow, Phys. Rev. D 59, 116008 (1999)

39. A.G. Cohen, S.L. Glashow, Phys. Rev. Lett. 97, 021601 (2006)

40. T. Jacobson, in From Quantum to Emergent Gravity: Theory and Phenomenology, Proceedings of Science, pp. QG-Ph 020 (2008)

41. P. Hořava, Phys. Rev. D 79, 0844008 (2009)

42. J. Rembieliński, Phys. Lett. B 730, 67 (2014)

43. G. Amelino-Camelia, Living Rev. Relativ. 13, 5 (2013)

44. G. Mackey, Induced Representations of Groups and Quantum Mechanics (W. A. Benjamin, Editore Boringhieri, Torino, 1968)

45. S. Weinberg, in Lectures on Particles and Field Theory, Lectures delivered at Brandeis Summer Institute in Theoretical Physics, ed. by S. Deser, K.W. Ford, vol. II (Prentice-Hall Inc, Englewood Cliffs, 1964), p. 405

46. R. Anderson, I. Vetharaniam, G.E. Stedman, Phys. Rep. 295, 93 (1998)

47. C. Lämmerzahl, Ann. Phys. 14(1-3), 71 (2005)

48. M. Jammer, Concepts of Simultaneity: From Antiquity to Einstein and Beyond (John Hopkins University Press, Baltimore, 2006)

49. S. Tanaka, Prog. Theor. Phys. 24(1), 171 (1960)

50. S. Schweber, An Introduction to Relativistic Quantum Field Theory (Row, Peterson and Company, Evanston, 1961)

51. N.N. Bogolubov, A.A. Logunov, I.T. Todorov, Introduction to Axiomatic Quantum Field Theory (W. A. Benjamin, Reading, 1975) 